\title{
Don't Panic: Recursive Interactions in a Miniature Metaworld
}

\author{
Andrew Bluff \\ andrew.bluff@uts.edu.au \\ Animal Logic Academy \\ University of Technology Sydney
}

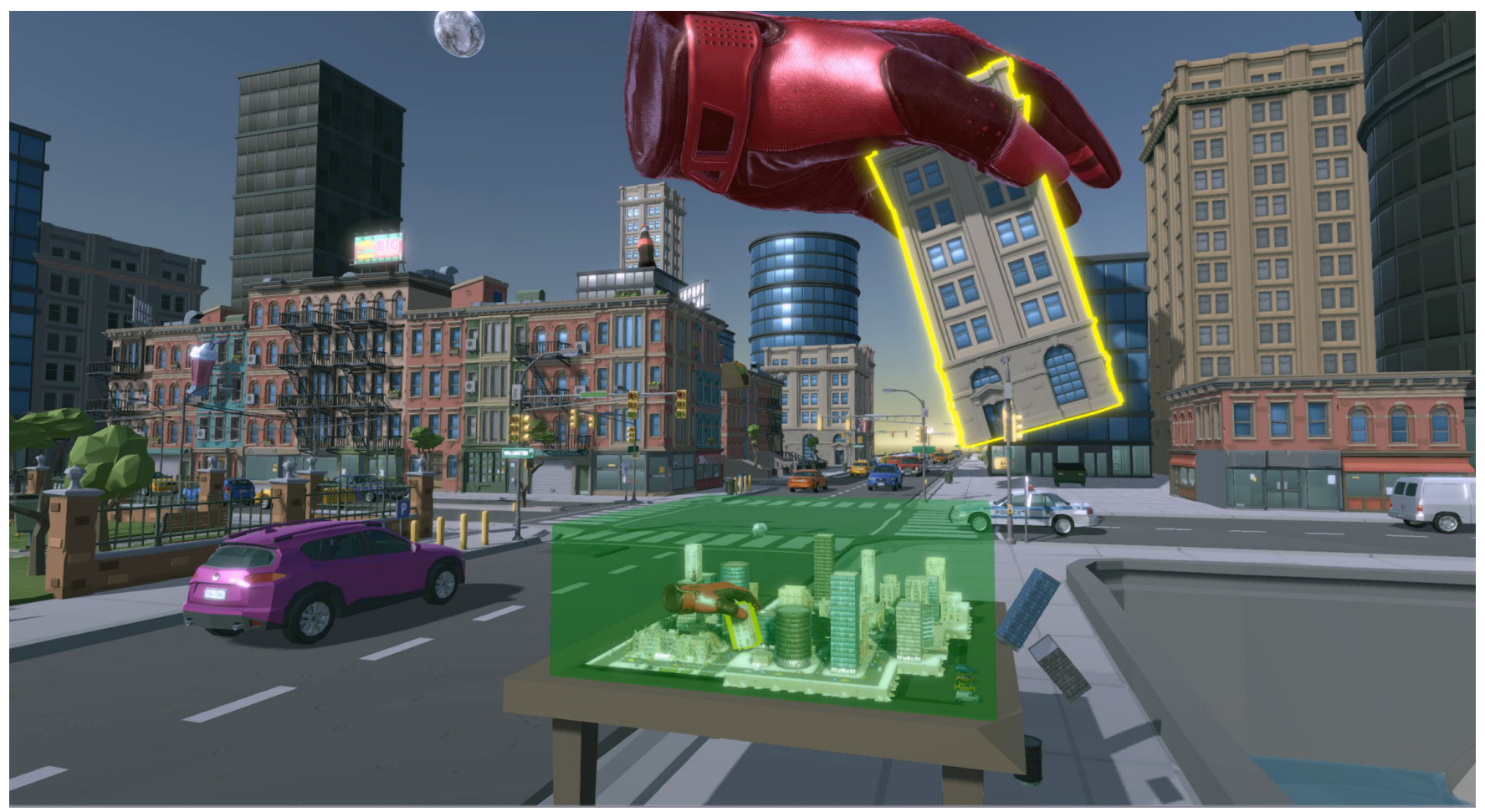

Figure 1: MetaCity - Grabbing a building from the miniature metaworld

\begin{abstract}
Metaworld is a new recursive interaction paradigm for virtual reality, where a miniature display (or 3D map) of the virtual world is presented to the user as a miniature model that itself lives inside the virtual world. The miniature model is interactive and every action which occurs on the miniature world similarly occurs to the greater virtual world and vice-versa. We implemented the metaworld concept in the virtual reality application MetaCity, a city designing sandbox where users can reach into a miniature model and move the cars and skyscrapers. Design considerations of how to display and interact with the miniature model are presented, and a technical implementation of the miniature world is described. The metaworld concept was informally and playfully tested in the MetaCity which revealed a number of novel interactions that enable the user to navigate quickly through large spaces, re-scale objects in the world and manipulate the very fabric of the world itself. These interactions are discussed within the context of four major categories - Experiential Planning, Interdimensional Transformations, Power of the Gods and Self Manipulation.
\end{abstract}

\section{CCS CONCEPTS}

- Human-centered computing $\rightarrow$ Interaction paradigms; Virtual reality.

\section{KEYWORDS}

metaworld, recursive interaction, virtual reality

\section{INTRODUCTION}

Virtual reality (VR) is an incredible tool for transporting the user into remote or uniquely fantastical worlds. This immersive technology aims to mimic the way in which the real world operates to provide the user with the maximum sense of being there (or 'presence') within a completely fabricated or virtual reality [Slater 2003]. Ideally, a virtual world provides an experience or ability which is not easily replicated in reality but which is still rooted in the basic laws of real-world physics that human beings tacitly understand. Despite being a concept since the 1960's [Burdea and Coiffet 2003], researchers and designers are still crafting and refining VR interaction models to facilitate super-human abilities that are simultaneously powerful and intuitive. We have seen virtual buttons and 
switches [Morimoto et al. 2007], floating two-dimensional displays [Ens et al. 2014] and numerous teleportation models [Bozgeyikli et al. 2016], all of which have their own unique sets of pros and cons in VR.

This paper proposes a new interaction paradigm where the user is transported to a virtual world that itself contains a miniature model of the very virtual world that they are inside. Imagine being able to shift furniture around a virtual room by playing with small miniature models of the furniture laid out on top of your desk - all while sitting inside that very room and watching it happen around you. In effect, you can become the designer or God of the own world that you inhabit.

After examining some related paradigms and concepts, we present MetaCity, an example VR application built to explore this recursive interaction paradigm. The technology used to create the application's miniature metaworld is described and a number of interactions facilitated by the concept are discussed.

\section{BACKGROUND}

\subsection{Science Fiction}

The concept of worlds nested inside other worlds is a common motif in Science Fiction. Many popular film and television programs reference a nesting of worlds including the multi-layered dream realities of Inception [Nolan 2010], the C18 beings living inside a railway locker in Men In Black 2 [Sonnenfeld 2002] (see Figure 2), Lisa's Petri-dish world in the Simpsons [Groening 1996], the miniature battery world of Rick and Morty [Dan Harmon 2018] and the giant supercomputer DeepThought (The Earth) in HitchHikers Guide to the Galaxy [Adams 2017]. These fictional worlds highlight a potential nesting of intelligent life operating on a miniature scale within our own reality and how we may be perceived as gods to these miniature civilisations. While these worlds are inspirational and often mind-bending, each miniature reality is its own cohesive universe. The metaworld concept differs from these popular examples through its inherently recursive nature, where the miniature world is a perfect holographic projection of the full-scale world and vice-versa - each reality cannot exist without the other because they are one and the same. A meta-physical 'wormhole' [Hawking 2009] where virtual spacetime is folded onto itself like the ancient Greek Dragon Ouroboros [Sheppard 1962] eating it's own tail to become an infinite self-fulfilling recursive loop.

\subsection{Computer Science}

Recursion is a powerful and commonly used tool in software design where certain algorithms can create meaning by successively calling upon themselves. In their seminal book on the foundations of computer science, Graham et al. describe recursion as where '...the solution to each problem depends on the solutions to smaller instances of the same problem' [Graham et al. 1989]. The self-referential miniature metaworld is a spatial and physical manifestation of this abstract notion of recursion.

\subsection{Inside out virtual reality}

Scale is an effective tool to manipulate within virtual and augmented reality (AR) applications and there are an increasing number of AR/VR apps providing miniature views in adjunct to the full-scale

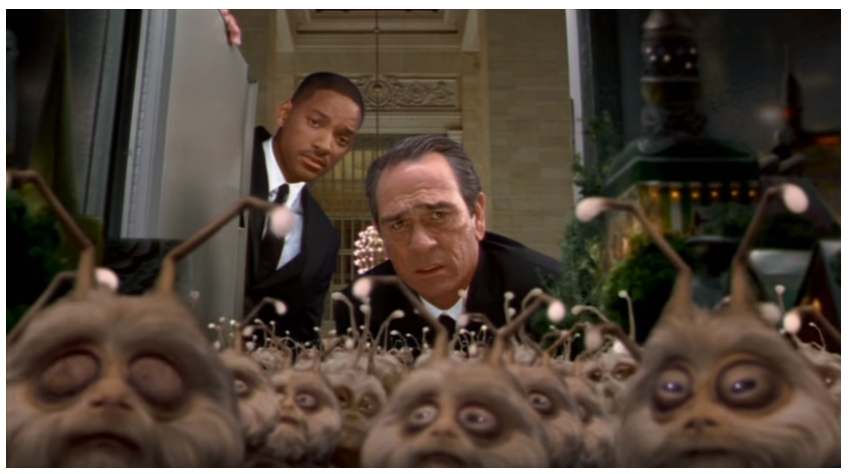

Figure 2: The locker world of Men In Black II ๑columbia Pictures

experience. In a bid to improve remote AR/VR collaboration, the Mini-Me [Piumsomboon et al. 2018] project applies a change in scale and perspective to a VR user's virtual body - shrinking their avatar to ensure that they always remain visible within the small field of view presented by the Hololens AR headset. The Magic Book [Billinghurst et al. 2001] is an augmented pop-up children's book that includes hand-held eye glasses with front facing cameras. The audience can look at the book through the glasses to see an augmented display of the book's world come to life. They can also choose to 'fly' into the book where the projection of the real world is disabled and participants become immersed inside the book in a lo-fi virtual reality. With a similar mixture of perspectives, the CALVIN system [Leigh et al. 1996] allows multiple networked users with a CAVE to view architectural models from either an 'inside-out' perspective (which mimics being inside the virtual building) or an 'outside-in' viewport (similar to looking at a miniature architectural model).

The Worlds in Miniature (WIM) VR project used a miniature copy of the life-sized virtual world as a method of interaction, allowing users to manipulate objects through the virtual miniature [Stoakley et al. 1995]. This example is closely related to the metaworld presented in this paper, although the implementation on the restricted 1990's VR devices prevented any full-sized navigation or interaction - all interaction was achieved via the miniature model. This restriction limited the experience, creating a scaled interactive view onto a full-sized virtual world, rather than a truly recursive meta-world.

\section{METAWORLD DISPLAY PARADIGM}

The concept of a recursive metaworld requires multiple instances of the same reality to be simultaneously present. The scale, rotation and/or position of each instance can differ to create multiple perspectives of the one unified reality. As this is a very abstract concept, when designing the metaworld display we looked at existing techniques from the real world which offer multiple perspectives of a unified reality.

\subsection{Real World Displays}

3.1.1 Paper Map. The most basic version of this concept is that of navigating a city with a paper map in hand. Looking around from a first-person perspective, we can see the buildings, streets, cars 
and people that surround us in our daily lives. If we look down at our map, we get an overview of a much larger section of the city and can plan our actions at a different scale. The map is another instance of the world in which we live, although it is merely a static 2D snapshot of the world we inhabit. You cannot interact with the map and affect the real world, and likewise anything that changes in the real world will not be reflected in the map.

3.1.2 Heads Up Display. Maps and displays which do mirror the inhabited world in real-time are commonly provided in computer games in the form of a "heads up display" (HUD). These HUDs are commonly presented in a static position of the users display and allows the player to gain an informational, navigational or tactical overview by displaying their current health status, weaponry status and/or the location of enemies and objectives. No matter which way you are facing in the gaming world, the HUD is always present in the same location of your display to provide extra information at a glance. The HUD concept was first introduced in real-world fighter planes where information such as altitude, roll/yaw, speed and fuel levels could be projected on the cockpit window allowing the pilot to view this information effectively superimposed onto the real world while keeping their heads up (ie not looking down at the instrumentation panel). Unlike a static map, the HUD display presents another instance of the world that is updated in real-time to mirror the events occurring in the 'real' world. However, these displays are non-interactive and provide two-dimensional data superimposed onto an inherently 3D world - a conflict which can create focal and attention issues that somewhat negate their realworld benefits [Fischer and Haines 1980; Tufano 1997].

3.1.3 Miniature Model. A model train set or miniature village display is another device commonly used to offer another perspective on the world. Unlike maps or HUDs, these are typically threedimensional models of a real world location presented at a miniature scale. They offer an overview of the real world from any angle, providing a unique three-dimensional perspective. These miniature models offer the ability for interaction where model trains or cars can be moved around the miniature world and can be used to plan for events. In the film Raiders of the Lost Ark [Spielberg 1981], for example, Indiana Jones interacts with a miniature model city to find the burial location of the Lost Ark. While miniature models allow for a three-dimensional and interactive perspective of a real world location, they do not synchronise with the real world in any way - events in the real-world are not reflected in the model and vice-versa.

\subsection{The Miniature Metaworld Display}

Virtual reality headsets allow us to create and explore fantastical realities, and we can use this technology to present multiple instances of the same reality, which are three-dimensional, interactive and synchronised in real-time. To keep the mind-bending possibilities of a recursive reality as simple and interactive as possible, we have primarily concentrated on the miniature world model. The implementation is conceptually simple - when you stand inside a virtual world (be it a house, city, forest or mountain) you will have a miniature model of the exact same location sitting on a small table in front of you.

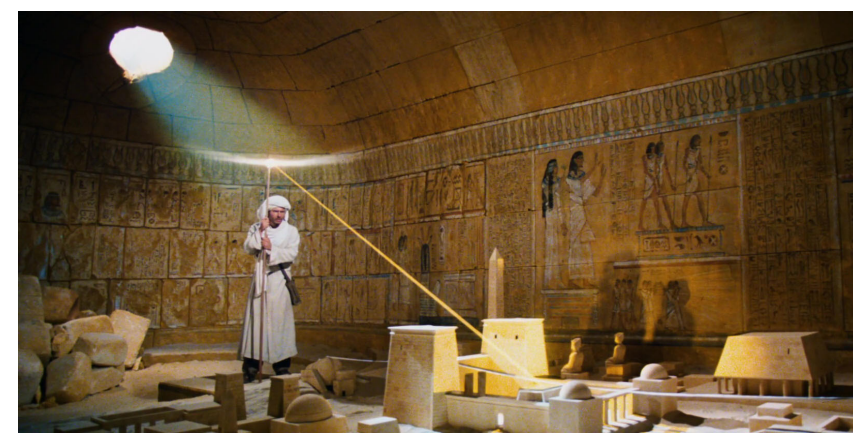

Figure 3: Indiana Jones navigates a miniature model city in Raiders of the Lost Ark $\odot$ Paramount Pictures

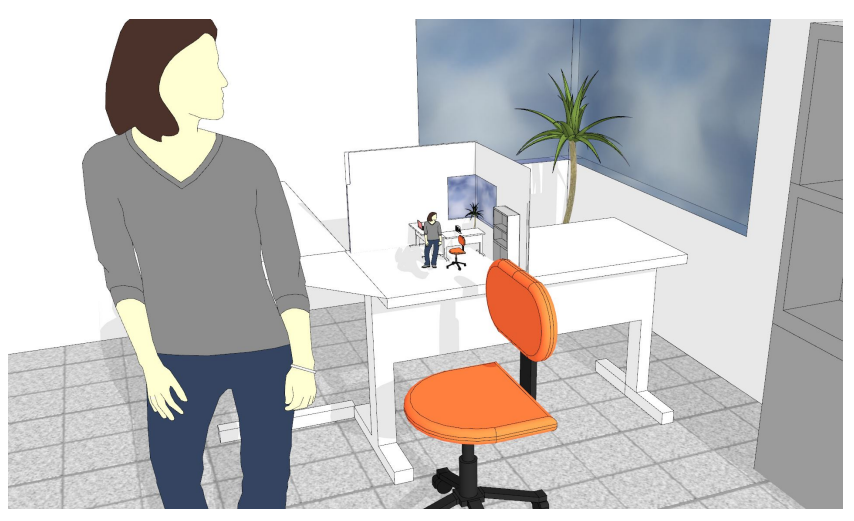

Figure 4: A virtual office with a miniature metaworld

To demonstrate how the display works, lets take the rather mundane example of using VR to portray a virtual office cubicle. You place the VR device on your head and as you look around the virtual room, you will see a desk, computer and an office chair. In the metaworld example, you will also find a miniature model of an office cubicle (see Figure 4). If you reach out and grab the chair from the full-scale virtual world with your controller, you can roll it across the room. If you now look down at the miniature model, you will also see that the miniature chair now rolling inside the miniature office. If you now reach inside the miniature model and move the miniature chair, you will see the full-scale version move in front of you. There is a direct consistency between what happens in the virtual world and what happens in the miniature model. Interaction with objects in the virtual cubicle can occur in either the full-scale virtual room or in the miniature model display.

The miniature model metaphor works well for room-scale VR where the user is presented inside a small room (like an office cubicle) and provides the user with an easy accessible 3D overview of the room that they are inside. However, as we expand the size of the virtual world, we find that the miniature model paradigm can become problematic. If we leave the virtual office and venture out onto a virtual street (commonly achieved in VR with a teleport mechanism) we can no longer see the miniature model - it is still sitting on a desk inside the office. We could scatter miniature model 
Table 1: Features of miniature display paradigms

\begin{tabular}{|c|c|c|c|c|c|}
\hline Paradigm & Overview & 3D & Interactive & Synchronised & Persistant \\
\hline Paper Map & Yes & - & - & - & If carried \\
\hline HUD & Yes & - & - & Yes & Yes \\
\hline Model & Yes & Yes & Yes & - & - \\
\hline Metaworld & Yes & Yes & Yes & Yes & Yes \\
\hline
\end{tabular}

tables all throughout the streets and landscapes of the virtual world but this would soon become cluttered and cumbersome.

To achieve the synchronisation and visual persistence of a HUD, the miniature world is presented sitting on top of a special small table in the virtual world. This small table is always in the same physical real-world location relative to the user's real-world position. As the user walks around in room-scale VR, they can walk around the virtual mini metaworld table and view the model from different perspectives. When the user teleports to a new location in the virtual world, their real-world position has not actually changed so the mini table display will remain in the very same location relative to the user, effectively teleporting alongside the user. Like a HUD, the miniature metaworld is always available and constantly reflects the changes in the larger virtual world, but like a miniature world it is three dimensional and easily interactable.

The miniature metaworld combines the features of all three realworld displays by providing a consistently synchronised view of the virtual world and being positioned in a consistent location in the user's real world (see Table 1).

To further clarify these concepts, we define the following terms to be used throughout this paper.

- Real World: This is the world that we inhabit - The room that you are standing in now.

- Virtual World: This is the world that you are transported to in a standard Virtual Reality experience. It is life-sized, but you can teleport around inside this virtual world while still remaining standing inside the real world.

- Miniature Metaworld: This is the miniature model of the virtual world that is on a table in front of you inside the Virtual World.

\section{TECHNICAL IMPLEMENTATION}

\subsection{MetaCity VR}

In order to investigate different styles of interaction made possible by the addition of a miniature metaworld, we created a sand-box urban environment that we call the MetaCity (see Figure 1). In this VR experience, the user can navigate the streets of a small city using standard VR walking and teleportation methods. The prototype virtual world includes city buildings, streets, cars, lampposts and some generic balls and blocks to play with. Importantly, it also includes a miniature metaworld so that interactions with the recursive environment can be tested.

The MetaCity VR app was developed using the Unity 2018 3D Game Engine, SteamVR plugin and the Polygon City Pack by Synty Studios. It was tested on the HTC VIVE system with head-mounted VR displays and 6DOF hand and controller tracking within a "roomscale" $2.5 \times 2.5 \mathrm{~m}$ space. The SteamVR plugin contains scripts to

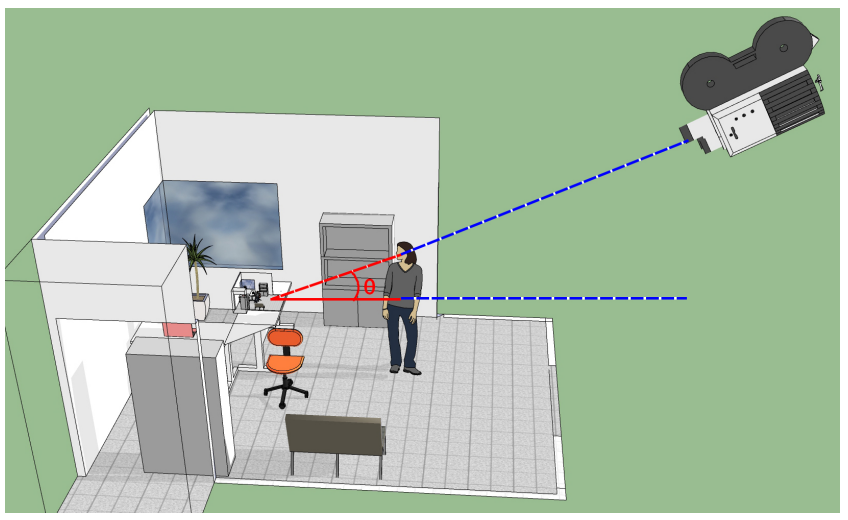

Figure 5: The metaworld camera follows the user as they circumnavigate the model

enable standard VR teleportation and provides simple grabbing, holding and throwing interactions.

\subsection{Virtual Display}

Rather than duplicating the world object and shrinking it down for the miniature model, the miniature metaworld is created by rendering the full-scale world and shrinking it down to size to ensure a $100 \%$ consistency at all times. To place this display into the virtual world, a flat plane was created at the position of the miniature display and is textured in real-time with the output of a special purpose in-world camera. This camera is maintained at a position which is consistent with the angle of the user to the display plane, but at a much larger distance and field of view (see Figure 5). This extra distance creates the illusion of a miniature model on the textured render plane. As the user moves around the plane, it automatically pivots to continually face the head of the user, preventing the perceptual sheer that occurs from walking around a thin rectangular plane in 3D space. To prevent the plane looking like a floating rectangular display, the metaworld render omits the surrounding skybox and uses a transparent texture to ensure that the silhouette of the miniature model is correctly depicted from all angles.

To duplicate the stereoscopic effect that is essential for portraying depth in VR, a separate textured plane and camera exists for each eye and the cameras are offset appropriately to mimic eyeseparation when peering at a miniature 3D model. This approach effectively creates a stereoscopic 3D VR display inside the greater virtual world. When the model is rendered at a sufficient scale and quality, this approach even produces a recursive display where the miniature model itself visibly contains a smaller miniature model display. There is no extra render or CPU cost for these smaller miniature displays, as the effect is similar to the $D r$ Who tunnel which is created by pointing a live video camera at a live video monitor. The dual cameras and floating render plane create a convincing 3D miniature display which plausibly sits as a real object inside the larger virtual world - displaying recursive versions of itself for the cost of one extra camera render. 


\subsection{Virtual Interactions}

An invisible box is placed around the floating display which acts as an interaction trigger. Any action that occurs inside this invisible box is treated as though it is operating on the miniature world Special scripts were created for the hand controllers so that when they passed through the invisible box, the controllers would become appropriately scaled up and would be operated remotely like a puppet. The scaling and dislocation of this puppet is continuously calculated in real-time to ensure that the hand controllers still appear to operate as normal inside the miniature world. When the hand is pulled out of the miniature world, the hand is scaled back to normal size and the puppet control is seamlessly removed without the user even noticing.

This script accounts for the hand controllers passing in and out of the miniature metaworld, but other objects also need to pass through the metaworld divide. The SteamVR interaction scripts operate by attaching objects onto the hand controllers when they are held. This has the beneficial side-effect where other world objects that are dragged into and out of the miniature world are automatically scaled and remotely puppeted alongside the hand controllers. This accounts for objects which are held but does not facilitate any unattached objects which fall (or are thrown) into the miniature world. To allow these unattached objects to seamlessly pass in and out of the miniature metaworld display, an additional script was added to detect when objects first enter the invisible interaction region. The script scales the object, its mass and current velocity vector appropriately and seamlessly teleports it into the larger fullscale world at the appropriate location to appear undisturbed from the perspective of the miniature metaworld.

The floating render plane technique and invisible interaction box implemented for the MetaCity present the user with a 3D miniature metaworld that can seamlessly transform objects in and out of the miniature space.

\section{INTERACTIONS AND PHENOMENON}

Having a recursive miniature metaworld inside a virtual world opens up a number of interaction possibilities. The design, implementation and playful testing of the MetaCity sandbox allowed the designers to conceptualise and trial many of these possibilities over a three month period. These interactions were found to operate under following four categories of Experiential Planning, Interdimensional Transformations, Power of the Gods and Self Manipulation.

\subsection{Experiential Planning}

A miniature metaworld provides the user with an overview perspective (useful for strategic planning and designing) while they simultaneously experience being present inside the very same world. The following interactions facilitate experiential planning.

5.1.1 Mapping, Planning and Navigation. Gaming and VR experiences often create an immersive world where users can be so absorbed in the moment that they can fail to plan their actions strategically - they fail to see the forest for the trees. Providing a miniature view of the user's immediate world allows an overview which can be useful for navigation and strategic planning tasks.
In addition, the first person view allows the user to experience a real-world scale depiction which is essential for providing the user a feeling of 'presence' in virtual reality. Providing these two perspectives simultaneously allows the user to see both the forest and the trees.

5.1.2 Architectural Manipulations. In order to fully immerse a user inside a virtual reality, the user should be able to interact with that world as they do the real world. The simplest and most common interaction in modern VR is the ability to pick up an object, rotate, place, throw and drop the object. The objects will mimic the realworld Newtonian laws of physics such as gravity and simple rigidbody collisions. These interactions are designed to immerse the user inside the virtual world but they provide very little control over the design of the virtual world itself. Providing these same interactions, but on a miniature model, allows the user to manipulate the world around them. They can easily move furniture, cars or even entire buildings around the world to shape it as they desire. It allows the user to become the architect of the very world that they inhabit.

\subsection{Interdimensional Transformations}

Interdimensional transformations are the interactions that arrive from passing objects through the interdimensional worm-hole from miniature to full-scale representation or vice-versa.

5.2.1 Resizing objects. Objects inside the miniature metaworld appear to be considerably smaller than the same object in the fullscale virtual world. If you reach into the miniature world and grab a skyscraper building from the model city and pull it out, you will have in your hands a miniature version of the building. The full-sized building in the full-sized virtual city around you has now been replaced by the miniature model building in your hands. The object has radically transformed in size by passing it through interdimensional worm-hole from miniature metaworld to the fullscaled virtual world. Any object that you pull out of the model world will be similarly transformed into miniature models - cars, houses, furniture or people will all become miniature versions. Conversely, any full-scale object will become giant when placed into the model world. Grab a golf ball and place it into the model and you now have an enormous monster ball crushing your office block from above. Put an ant into the model and you will have a large creepy looking six-legged horse to ride around.

5.2.2 Falling out of world. To create a completely recursive depiction of the world, the boundaries of the miniature metaworld should also mirror the boundaries of the larger virtual world. This means that if your miniature world is sitting on a table, the larger virtual world should also be sitting on an even larger table. When you walk or teleport to the bounds of your virtual world (city, office cubicle or forest etc), you should be able to peer over and see the giant table on which your virtual world is sitting. Any objects that fall (or are thrown) off the edge of the full scale virtual world will simply appear back as miniature objects which have fallen off the miniature metaworld table back into the larger virtual world.

5.2.3 Gravity, physics and collisions. In addition to resizing, when objects are dropped or thrown in, out or through the miniature metaworld, we need to account for a change in the laws of physics. 


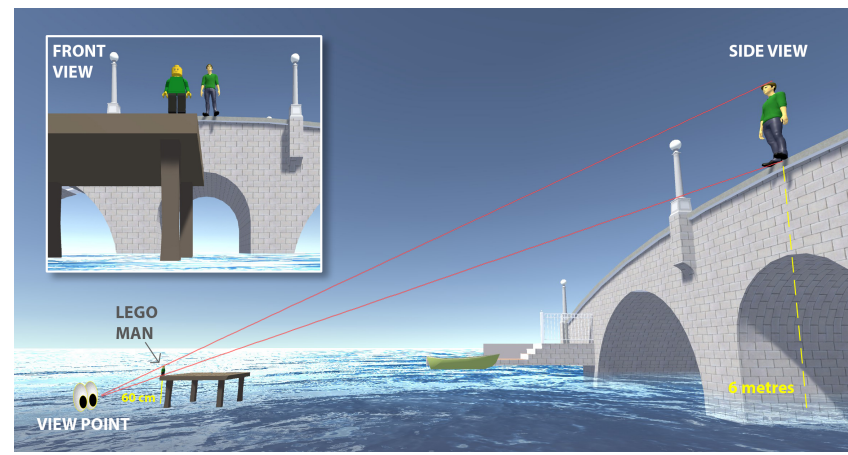

Figure 6: A LEGO man viewed at the same size as a full-scale human will hit the ground faster

The mass of an object needs to scale up or down with the size, and the speed at which Newtonian physical forces act also needs to scale. Forces like gravity work very differently on a miniature model than they do on a full sized reality. For example, Figure 6 shows a miniature LEGO man placed a small table very close to the user's eyes such that the LEGO man appears to be the same size as a real man standing on top of a distant bridge. When the Lego man is pushed at the exact same time that the real man jumps, the LEGO man will fall the $60 \mathrm{~cm}$ to the ground very quickly while the real person will take much longer to drop to $6 \mathrm{~m}$ down to the water below. This creates a minor conflict - the LEGO man and the real person are in fact the exact same person viewed from different perspectives in the metaworld, but gravity should operate differently on them depending on which reality they are perceived to be in. There are three major paradigms for handling this situation:-

- Virtual world dominant: In this scenario the physics is all based on the full-scale depiction of the virtual world. Gravity and collisions will occur as they do in the real world, but the miniature metaworld display will look like a crystal ball viewport of the world rather than a true miniature model which actually exists inside the real world.

- Focus first: In this scenario all of the objects in the world react to physics according to the laws of the reality of the world that the user is current looking at. If your head is up and you are looking at the virtual world around you, then real-world full-scale physics will occur. However, if you are currently looking at the miniature metaworld, then gravity and collisions will be sped up so that it reacts as though everything is living inside a miniature world (i.e. the LEGO man on the table falling to the ground). This has the benefit of the world behaving as the user will most likely expect when focusing on either the real world or the miniature world. This effect, however, may break down if the user looks at both views simultaneously or switches focus between the two displays quickly while collisions and gravity are occurring. In such a situation, varying degrees of focus and a hysteresis could be added so that the world's physical laws smoothly morph from one situation to the other. This could create a simplistic 'bullet time' effect where collisions gradually slow down or speed up as you focus more or less on the miniature metaworld display. Focus becomes an effective interaction style allowing the user to control the very laws of physics.

- Origin of interaction: With this paradigm, each object contains a notion of their own laws of physics. If the objects are activated (thrown, dropped or bashed) by another object then it will acquire the laws of physics from the initiating object. The user's controllers will inherit the physics laws to match the world that they are currently in. Reusing our LEGO man example, it means that if the user pushes that man from the bridge in the full-scale virtual world, they will plummet to the river below at real-world speeds. However, if you stick your hand into the miniature metaworld and push the miniature (LEGO) version of the that man then he will fall very quickly as you would expect a miniature model to fall. This paradigm places the shift of focus from a visual one (focus first) to that of a physical interaction as was therefore the method selected for the MetaCity.

\subsection{Power of the Gods}

The interactions in the Power of Gods category rely on the user manipulating the model of the miniature world itself, providing the user with God-like powers over the world within (and therefore their own full-scale virtual world).

5.3.1 Tilting the world. Imagine picking up a model city packed full of model cars. As you begin tilting the model, all of the cars would slowly roll off the streets and end up falling off the model. The same effect occurs with the miniature metaworld model, where all of the objects around you roll around depending on what direction the miniature model is tilted. After trialling this effect in the MetaCity, it soon became apparent that rotating the user or their perspective in VR can easily lead to motion sickness. In contrast, locking the user to the floor (as though they are wearing suction boots) and tilting the gravitational forces of the virtual world is preferable as it produces the interesting rolling effects without the vestibular manipulations that can often lead to sickness.

5.3.2 Shaking the world. This is an extension of tilting the world. Picking up the miniature world and shaking or throwing it around can act like a snow-globe and cause havoc in the full-scale virtual world as objects fly chaotically throughout the entire world like giant snowflakes. This phenomenon is an extension of the tilting effect whereby both direction and scale of gravity are affected by the shaking motions. The harder you shake, the stronger gravity forces become and the more things fly around the room.

5.3.3 Bending the world. If we exchange the rigid base of our virtual miniature model for a semi-flexible cardboard structure, we can bend the base of the miniature model by picking it up in two hands and applying opposing forces. As the base of the model warps in the user's hands, the entire surface of the virtual world will similarly bend like a catastrophic earthquake. Further warping can create Escher-like mutations on the real-world geometry similar to the world bending scenes of 'Doctor Strange' or 'Inception'.

5.3.4 Blowing into the mini world. Many consumer VR headsets contain microphones that can be used to detected when a user 


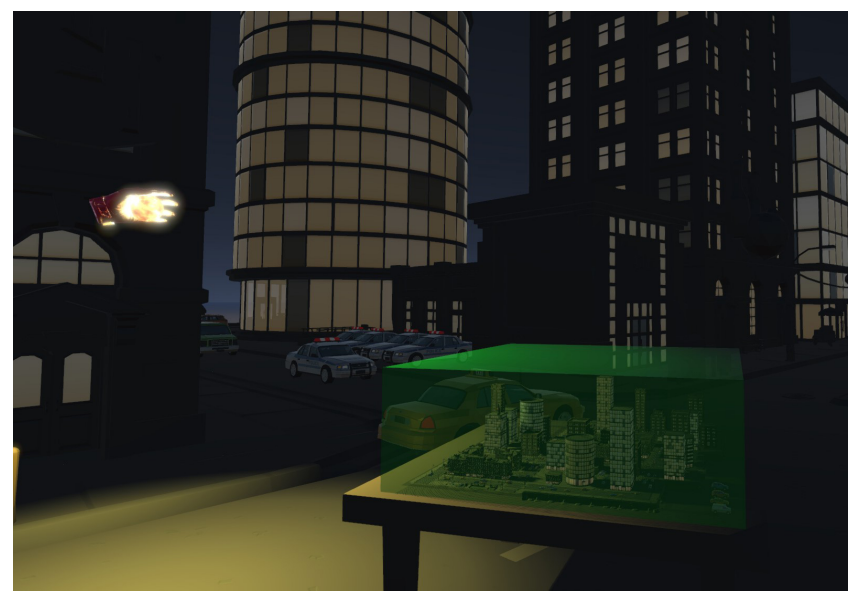

Figure 7: Holding a miniature sun in the MetaCity

physically blows air out of their mouth. While blowing in a fullscale virtual world almost never has any meaningful effect, blowing onto a miniature metaworld can create enormous 'God-like' winds that act as huge forces on the full-scale virtual world. This creates another method for shifting items around in a similar (but possibly more intuitive) fashion to tilting the world.

5.3.5 Moving the sun. Virtual worlds mimic their real world counterparts as closely as possible and this extends to lighting where sunlight systems are commonplace VR worlds. While the Sun is unreachable in a standard VR world, a miniature metaworld lets the user reach into the model and pluck the sun right out of the sky. The user can now move the day/night cycle to any position by intuitively grabbing and moving the Sun as they would any other object. Dragging the sun out of the miniature model altogether draws the virtual world in darkness and leaves the user holding a miniature lantern (see Figure 7). While still interactive, the gravity was disabled on the MetaCity's Sun in so that it could hang effortlessly in the sky without plummeting to the ground.

\subsection{Self Manipulation}

In addition to the God-like powers that a miniature metaworld provides, there is also the realisation that the user themselves is also a part of the miniature world. This allows for the user to have god-like powers over their own virtual body and opens up some more interesting interactions.

5.4.1 Dislocation of limbs. Reaching your hand into the miniature model and seeing it become the giant hand of god is quite interesting in its own right (see Figure 1). Imagine stamping on the miniature model and seeing a giant Monty-Python style foot crushing down from above. The user is disconnected from their giant limbs in terms of both scale and location, but they are obviously still very connected to the users own body and movements.

5.4.2 Self Relocation. VR features standard methods to move around that include teleportation and physically walking (up to the boundaries of the room). The miniature metaworld model provides a novel way to navigate the space where a user can reach into the model and literally pick themselves up and place themselves anywhere inside the room. This is a very intuitive and quick way to navigate the world, but can initially produce feelings of motion sickness. During testing, these ill feelings subsided after three or four attempts and the designers could navigate large distances with ease.

5.4.3 Inter-Dimensional Teleportation. Teleportation is a quick way to move short distances in VR, but if we add the ability to use teleportation on the miniature metaworld model, then the user can teleport great distances with a single click. Select where you want to go on the miniature map and you are instantly transported there. More interestingly, if we truly follow the recursive metaworld paradigm, you should be transporting into the model itself. This creates the ability to scale the size of the user themselves within the virtual world and can affect gravity and the laws of physics as they morph to operate in the same manner as a miniature model. We now have an "eat me" / "drink me" portal device to shrink and expand the user as they teleport through the metaphorical rabbit-hole.

\section{FUTURE RESEARCH}

\subsection{Unexplored Interactions}

The interactions presented in this paper were implemented and play-tested by the designers in the prototype VR app, MetaCity. These only represent a mere sample of the possibilities opened up by the metaworld paradigm. Upon future research the authors would like to explore:-

- Floppy world: Extending the bending the world concept by making the base of the miniature model into a soft and floppy fabric could facilitate the terraforming of mountains and valleys.

- Sound manipulation: Adding snippets of sound to virtual objects would create an interesting sound manipulation sandbox as samples are time-stretched and re-pitched as objects are rescaled.

- Falling from the virtual world: When the user falls out of the virtual world, they would end up falling out of the miniature model at a miniature scale.

- Climbing out of the miniature model: What happens when the user climbs the walls of the virtual world to break free of their own miniature model?.

- A falling metaworld: What should logically happen when the user throws the miniature metaworld off the side of the virtual world?

- Duplication: If two miniature metaworlds existed in the one experience, any objects which fall out of the virtual world should fall out of both of the miniature worlds, effectively duplicating the object.

- Cascading Loops: Multiple metaworlds would also facilitate complex recursive loops where the miniature worlds cascade to other miniature worlds creating an infinite waterfall loop of duplication and scale manipulation.

\subsection{Potential Applications}

The MetaCity example was created as a sandbox to play with the metaworld concept and play-test the interaction ideas it presented. Future research could apply the concept to other VR applications 
and formally evaluate how real world users respond to the metaworld paradigm in the diverse areas such as:-

- Architectural and Interior Design: The ability to build your surroundings on a miniature scale while actually being inside that space enables the designer to experience and plan simultaneously without any context switching.

- City Planning: The power to move the Sun provides a quick and intuitive way to examine how tall buildings will interplay with shadowing in different times of day and year, and being inside the city while testing facilitates a very experiential design approach.

- Game Design: The ability to build a game world while actually playing a game in VR may once again reduce context switching and provide an effecient design environment.

- Action Gaming: The device allows a blend of strategic and first person action gaming in the one seamless environment. The self-relocation ability of being able drag yourself anywhere around the world adds a new way to navigate large open world environments.

- Puzzle Gaming: The mind bending physics of changing the scale of objects and messing with god-like powers of bending, blowing and tilting the miniature world would suit a 'Portal'- style of VR puzzle game.

- Historical Education: Gain an insight into how ancient civilisations structured their cities or dwellings and how these large scale organisational structures impact the actual living spaces of its inhabitants and vice-versa.

- Data Simulations: Navigate or manipulate large data sets from an overview perspective while simultaneously examining data points from and intimate point of view. Different informational traits could be displayed at different scales and intuitively traversed.

- Medical Training: Practice surgery on virtual patients while seeing the effect of your actions from inside the body. Witness how a virus can attack on a cellular level, while seeing an overview of the body as the virus spreads.

- Electronic music: A metworld music app could allow a musician to create, edit and rescale music at a compositional, musical note, textural or microsound [Roads 2004] level simultaneously.

- Physics Explorer: Users could explore real-world physical phenomena such as galaxies, stars, atoms and quasars from different perspectives.

- Recursion Sandbox: The metaworld could be used to teach concepts of recursion to introductory maths and computing students of all ages.

\section{CONCLUSION}

The metaworld provides a unique perspective where you can access your own virtual world both from within and looking down on an overview at the same time. This unique dual perspective provides some mind twisting effects and interesting interaction opportunities such as Experiential Planning, Interdimensional Transformations, Power of the Gods and Self Manipulation. The interactions and effects presented in this paper were uncovered through the implementation and playful testing of MetaCity, a virtual reality urban sandbox. The desire to make the miniature world behave as an overview portal/viewport into the current world while simultaneously acting like a miniature model situated inside the greater virtual world creates some unique conflicts in the basic laws of physics. These conflicts offer further potential for unique interaction styles where the user can be given a certain amount of control over the ways that gravity and physics behave in real-time.

Given the ubiquity and utility of heads up displays (HUDs) in videogames, blueprints for town planning and maps for personal navigation, the miniature metaworld display represents the initial iteration of an interactive paradigm which could become an essential tool for many varied virtual reality experiences in the future.

\section{ACKNOWLEDGMENTS}

Thanks to the members of UTS Animal Logic Academy Research and Creativity and Cognition Studios for their insightful comments and editorial assistance.

\section{REFERENCES}

Douglas Adams. 2017. The Hitchhiker's Guide to the Galaxy Omnibus: A Trilogy in Five Parts. Vol. 6. Pan Macmillan.

Mark Billinghurst, Hirokazu Kato, and Ivan Poupyrev. 2001. The MagicBook: a transitional AR interface. Computers \& Graphics 25, 5 (2001), 745-753.

Evren Bozgeyikli, Andrew Raij, Srinivas Katkoori, and Rajiv Dubey. 2016. Point \& teleport locomotion technique for virtual reality. In Proceedings of the 2016 Annual Symposium on Computer-Human Interaction in Play. ACM, 205-216.

Grigore C Burdea and Philippe Coiffet. 2003. Virtual reality technology. John Wiley \& Sons.

Justin Roiland Dan Harmon. 2018. Rick and Morty.

Barrett Ens, Juan David Hincapié-Ramos, and Pourang Irani. 2014. Ethereal planes: a design framework for 2D information space in 3D mixed reality environments. In Proceedings of the 2nd ACM symposium on Spatial user interaction. ACM, 2-12.

Edith Fischer and Richard F Haines. 1980. Cognitive issues in head-up displays. (1980).

Ronald L Graham, Donald E Knuth, Oren Patashnik, and Stanley Liu. 1989. Concrete mathematics: a foundation for computer science. Computers in Physics 3, 5 (1989), 106-107.

Matt Groening. 1996. Simpsons: Treehouse of Horror VII.

Stephen Hawking. 2009. A brief history of time: from big bang to black holes. Random House.

Jason Leigh, Andrew E Johnson, Christina A Vasilakis, and Thomas A DeFanti. 1996. Multi-perspective collaborative design in persistent networked virtual environments. In Virtual Reality Annual International Symposium, 1996., Proceedings of the IEEE 1996. IEEE, 253-260.

Kazuhiro Morimoto, Chiyomi Miyajima, Katsunobu Itou, and Kazuya Takeda. 2007. A virtual button interface using fingertip movements. In 2007 International Conference on Machine Learning and Cybernetics, Vol. 4. IEEE, 2089-2093.

Christopher Nolan. 2010. Inception.

Thammathip Piumsomboon, Gun A Lee, Jonathon D Hart, Barrett Ens, Robert W Lindeman, Bruce H Thomas, and Mark Billinghurst. 2018. Mini-me: an adaptive avatar for mixed reality remote collaboration. In Proceedings of the $2018 \mathrm{CHI}$ conference on human factors in computing systems. ACM, 46.

Curtis Roads. 2004. Microsound. MIT press.

Harry J Sheppard. 1962. The Ouroboros and the Unity of Matter in Alchemy: A Study in Origins. Ambix 10, 2 (1962), 83-96.

Mel Slater. 2003. A note on presence terminology. Presence connect 3, 3 (2003), 1-5. Barry Sonnenfeld. 2002. Men In Black 2.

Steven Spielberg. 1981. Raiders of the Lost Ark.

Richard Stoakley, Matthew J Conway, and Randy Pausch. 1995. Virtual reality on a WIM: interactive worlds in miniature. In CHI, Vol. 95. 265-272.

Daniel R Tufano. 1997. Automotive HUDs: The overlooked safety issues. Human factors 39, 2 (1997), 303-311. 